\title{
Combinatory strategy for characterizing and understanding the ethanol synthesis pathway in cyanobacteria cell factories
}

\author{
Guodong Luan ${ }^{1,2+}$, Yunjing $\mathrm{Qi}^{4 \dagger}$, Min Wang ${ }^{1,3}$, Zhimin Li ${ }^{1,2,6}$, Yangkai Duan ${ }^{1,3,5}$, Xiaoming Tan ${ }^{1,2}$
} and Xuefeng $L^{1,2^{*}}$

\begin{abstract}
Background: Photosynthetic production of chemicals and fuels by recycling $\mathrm{CO}_{2}$ in cyanobacteria is a promising solution facing energy shortage and resource declination. Ethanol is an attractive and demonstrative biofuel product, and ethanol synthesis in cyanobacteria has been achieved by assembling of a pathway consisting of pyruvate decarboxylase (PDCZm) and alcohol dehydrogenase II (s/r1192). For enabling more powerful ethanol photosynthetic production, an optimized and balanced catalyzing route was required. In this work, we provided a paradigm for systematically characterizing and optimizing the PDCzm-sIr1192 pathway from engineered cyanobacteria strains, combining in vitro reconstitution, genetic engineering and feeding-cultivation.
\end{abstract}

Results: We reconstituted the PDCzm-sIr1192 pathway in vitro and performed specific titration assays for enzymes, substrates, cofactors, and metal ions. In the in vitro system, $K_{50}$ of PDCzm was $0.326 \mu \mathrm{M}$, with a $V_{\max }$ of $2.074 \mu \mathrm{M} / \mathrm{s}$; while for slr1 192, the values were $0.109 \mu \mathrm{M}$ and $1.722 \mu \mathrm{M} / \mathrm{s}$, respectively. Titration response discrepancy indicated that PDCzm rather than sIr1192 was the rate-limiting factor for ethanol synthesis. In addition, a 4:6 concentration ratio of PDCzm-slr1 192 would endow the reaction with a maximal specific catalytic activity. Titration assays for other components were also performed. $K_{m}$ values for NADPH, pyruvate, TPP, $\mathrm{Mg}^{2+}$ and acetaldehyde were $0.136,6.496,0.011$, 0.104 , and $0.393 \mathrm{mM}$, respectively. We further constructed Synechocystis mutant strains with diverse PDCzm-slr1192 concentrations and ratios, and compared the growth and ethanol synthesis performances. The results revealed that activities of PDCzm indeed held control over the ethanol generation capacities. We performed pyruvate-feeding treatment with the newly developed Syn-YQ4 strain, and confirmed that improvement of pyruvate supply would direct more carbon flow to ethanol formation.

Conclusions: We systematically characterized and optimized the PDCzm-slr1192 pathway in engineered cyanobacteria for ethanol production. Information gained from in vitro monitoring and genetic engineering revealed that for further enhancing ethanol synthesis capacities, PDCzm activities needed enhancement, and the PDCzm-sIr1192 ratio should be improved and held to about 1:1.5. Considering actual metabolites concentrations of cyanobacteria cells, enhancing pyruvate supply was also a promising strategy for further updating the current ethanol photosynthetic cell factories.

Keywords: In vitro reconstitution, Steady-state analysis, Ethanol, Cyanobacteria, Metabolic engineering

\footnotetext{
*Correspondence: Ivxf@qibebt.ac.cn

${ }^{\dagger}$ Guodong Luan and Yunjing Qi have contributed equally to this work

${ }^{2}$ Shandong Provincial Key Laboratory of Synthetic Biology, Qingdao Institute of Bioenergy and Bioprocess Technology, Chinese Academy of Sciences, No. 189 Songling Road, Qingdao 266101, China

Full list of author information is available at the end of the article
} provided you give appropriate credit to the original author(s) and the source, provide a link to the Creative Commons license, and indicate if changes were made. The Creative Commons Public Domain Dedication waiver (http://creativecommons.org/ publicdomain/zero/1.0/) applies to the data made available in this article, unless otherwise stated. 


\section{Background}

Globally increasing energy demands and irreversibly declining fossil resources are driving the development of efficient and sustainable production routes for chemicals and fuels [1]. Photosynthetic cell factories directly channeling solar energy and $\mathrm{CO}_{2}$ into aimed products are generally accepted as one of solutions [2-4]. Cyanobacteria have displayed great potentials to be a promising photosynthetic chassis, with the characteristics of simple structure, rapid growth, and convenient genetic manipulations [5, 6]. Assembling, inserting, and fine-tuning of the heterologous and endogenous enzymes in cyanobacteria has enabled photosynthetic generation of multiple products, including alcohols, ketones, alka(e)nes, organic acids, and sugars [7-13].

Ethanol is the first reported and most representative cyanobacteria-based photosynthetic biofuel product. In 1999, Deng and Coleman endowed Synechococcus sp. PCC7942 with ethanol synthesis capacities by introduction of a 2-step metabolic pathway from Zymomonas mobilis, consisting of pyruvate decarboxylase (PDCzm encoded) and alcohol dehydrogenase II ( $A D H z m$ encoded) [8], which has also been widely applied in many other ethanol-producing microbial cell factories [1416]. ADHzm was further replaced with a native alcohol dehydrogenase slr1192 from Synechocystis sp. PCC6803 showing improved NADPH-preference [17] and catalytic efficiency $[17,18]$ in cyanobacteria. Ever since, many strategies and methods have been adopted for efficiently rerouting the carbon fixed in Calvin cycle to ethanol formation in engineered cyanobacteria strains, including deletion or weakening of competitive pathways, improvement of photosynthesis activities, strengthening of precursor supplies, and engineering of ethanol-tolerance, while catalytic activities of the core pathway (PDC-ADH) proved to be the most essential factor determining ethanol synthesis capacities [18-23]. For developing more powerful ethanol photosynthetic cell factories, an optimized and balanced PDC-ADH catalytic process would be the premise, which requires systematic characterization and comprehensive understanding of the metabolic pathway.

In this work, we adopted a systematic strategy combining in vitro reconstitution, genetic engineering and feeding-cultivation to characterize and understand the PDCzm-slr1192 pathway in ethanol synthesis strains of Synechocystis sp. PCC6803 (Synechocystis hereafter). For monitoring and unveiling the kinetic characteristics and system properties of the conversion process from pyruvate to ethanol, we reconstituted the PDCzm-slr1192 pathway in vitro and performed quantitative titrations for each specific component, including enzymes, substrate, and cofactors. Accurate contribution assessments for each factor of the ethanol synthesis process indicated that improving the PDCzm-slr1192 concentration ratio and enhancing pyruvate supply might be promising engineering strategies for promoting ethanol photosynthetic production. For validation, we regulated the $P D C z m$ and slr1192 copy numbers in Synechocystis to disturb the enzyme concentration ratios, and took pyruvate-feeding strategies to enhance the substrate supply. Calculations of enzymatic activity, cell growth, ethanol synthesis, and carbon partition ratio of the Synechocystis strains matched well with the predictions from in vitro system, indicating that our results could serve as useful guidance for updating the current ethanol photosynthetic cell factories.

\section{Results}

In vitro reconstitution and quantitative titrations of PDCzm and sIr1192 for ethanol synthesis

As shown in Fig. 1, the conversion process in engineered Synechocystis strains from pyruvate to ethanol was catalyzed by two enzymes, PDCzm catalyzing the decarboxylation of pyruvate to acetaldehyde, which would be further converted to ethanol by slr1192. During the process, equal moles of NADPH would be consumed by slr1192 when ethanol was generated, so the consumption speeds of NADPH could be measured to evaluate the reaction velocities.

PDCzm and slr1192 were expressed under T7 promoter in Escherichia coli BL21 (DE3) and purified individually. As an initial reference system, $0.05 \mu \mathrm{M}$ PDC and $0.05 \mu \mathrm{M} \operatorname{sir} 1192$ were added to a $500 \mu \mathrm{l}$ reaction system containing $100 \mathrm{mM}$ Tris- $\mathrm{HCl}, 10 \mathrm{mM}$ pyruvates, $0.2 \mathrm{mM}$ $\mathrm{NADPH}, 10 \mathrm{mM} \mathrm{MgCl}$ and $0.1 \mathrm{mM}$ TPP. Absorbance of the system at $340 \mathrm{~nm}$ was measured with a Beckmancoulter DU-800 spectrophotometer, and the decreasing speed of NADPH was converted to the reaction velocities based on the absorptivity of NADPH, $6.22 \times 10^{3} \mathrm{l} /$ $\mathrm{mol} / \mathrm{cm}$. For the initial reference system, the velocity was $0.452 \pm 0.04 \mu \mathrm{M} / \mathrm{s}$.

We further performed in vitro titrations for PDCzm and $\operatorname{sir} 1192$ to assess the specific contributions from each enzyme and identify the rate-limiting step for ethanol synthesis. Concentrations of one enzyme were increased to $0.1,0.2,0.5,1,1.5,2$, and $3 \mu \mathrm{M}$, while that of the other one was kept at $0.05 \mu \mathrm{M}$. As shown in Fig. 2a, titrations of PDCzm and slr1192 revealed markedly different responses. According to a previously reported calculation method [24], initial reaction velocities versus enzyme concentrations plots were fitted with the Michaelis-Menten model, while the substrate were replaced with enzyme $\left(V=V_{\max } \times E /\left(K_{50}+E\right)\right.$, here $K_{m}$ value for substrate was replaced with $K_{50}$ for enzyme). $K_{50}$ value of PDCzm was about $0.326 \mu \mathrm{M}$, with a $V_{\max }$ 


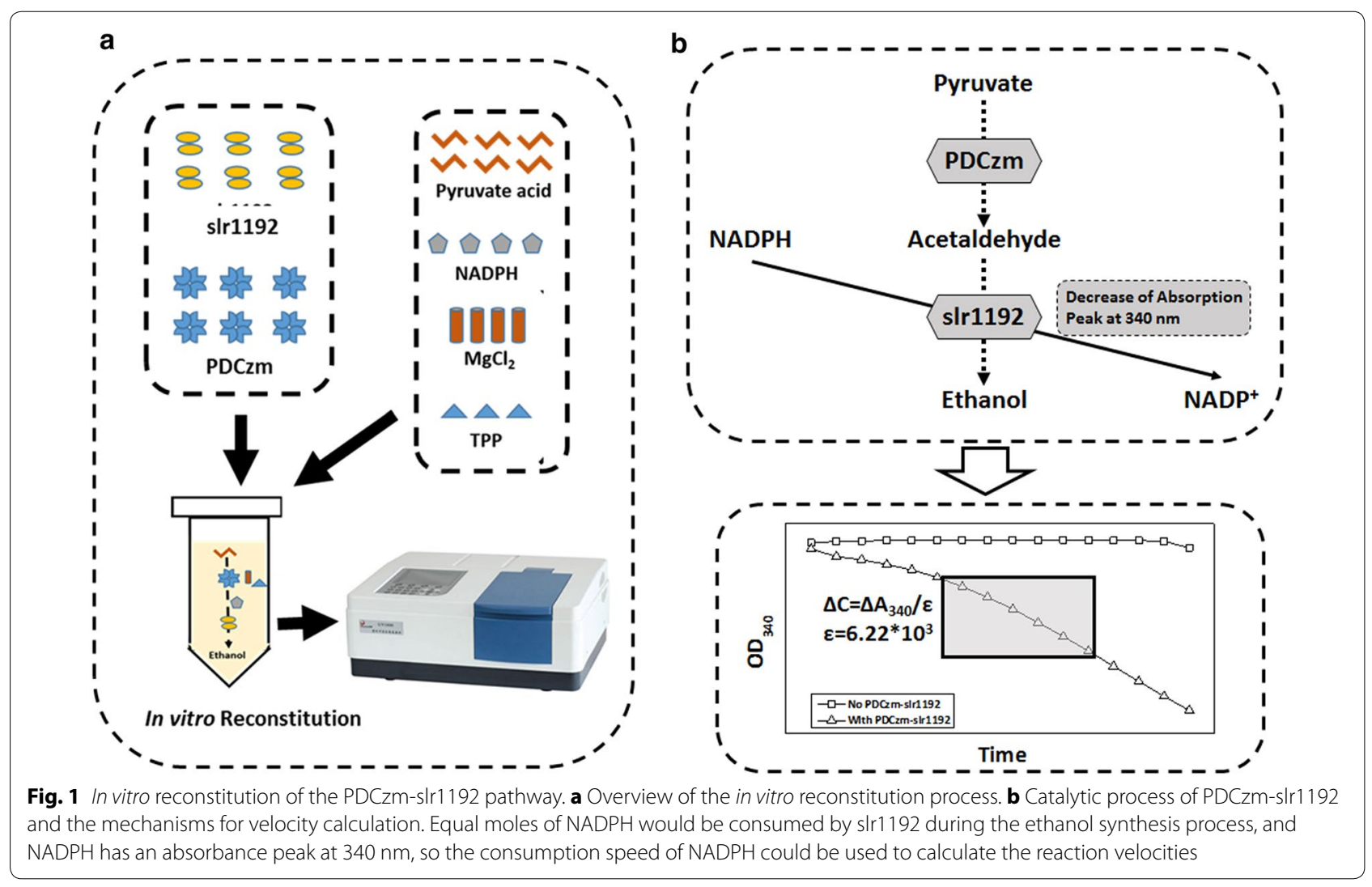

value of $2.074 \mu \mathrm{M} / \mathrm{s}$, while $K_{50}$ and $V_{\max }$ for slr1192 were $0.109 \mu \mathrm{M}$ and $1.722 \mu \mathrm{M} / \mathrm{s}$, respectively. The relatively higher $K_{50}$ and $V_{\max }$ of PDCzm comparing with slr1192 indicated that for the initial reference system, PDCzm rather than slr1192 played as the main rate-limiting factor.

We further analyzed the specific activities dynamics of the PDCzm-slr1192 total proteins during the titration processes. As shown in Fig. 2b, for PDCzm titration, with increasing of the total protein concentrations, specific activities of the PDCzm-slr1192 system kept decreasing from 0.089 to $0.01 \mu \mathrm{M} /(\mathrm{s} \mathrm{mg} / \mathrm{l}$ protein). As for the slr1192 titration, a peak with the highest specific activities $[0.13 \mu \mathrm{M} /(\mathrm{s} \mathrm{mg} / \mathrm{l}$ protein $)]$ appeared when the slr1192 concentration reached $0.1 \mu \mathrm{M}$, indicating that the most economical and balanced concentration ratio of PDCzm-slr1192 might be around 1:2.

\section{Optimization of the PDCzm-sIr1192 ratio for the in vitro assay system}

As for construction of microbial cell factory, improving and optimizing expression levels of the enzymes in the metabolic pathway was always an important strategy; however, concentrations of specific enzymes could not be endlessly increased. A balanced and economical concentration ratios between diverse metabolic components might be a more promising choice for in vivo pathway engineering. Thus we regulate the concentration ratios of PDCzm and slr1192 from 0:10 to 10:0 in the in vitro system with total protein concentrations of $10 \mathrm{mg} / \mathrm{l}$.

As shown in Fig. 2c, when the concentration ratio of PDCzm-slr1192 reached 4:6 (mole ratio 1:2.5), reaction velocities of the in vitro system taking NADPH as the cofactor were maximized. That result perfectly matched the response of slr1192 titration, in which reaction system with a 1:2 mole ratio of PDCzm-slr1192 showed the highest specific activities. We also measured the velocity dynamics for diverse PDCzm-slr1192 concentration ratios in in vitro system taking $\mathrm{NADH}$ as the cofactor. As shown in Additional file 1: Figure S1, when $0.2 \mathrm{mM}$ $\mathrm{NADH}$ rather than NADPH was added to the in vitro system, reaction velocities decreased dramatically, due to the low preference of slr1192 for NADH [25]. Meanwhile, the most efficient concentration ratio moved to be $3: 7$ instead of $4: 6$, and that might be caused by that the decrease of slr1192 activities in the NADH environments disturbed the previously generated balance. Considering that cellular concentrations of NADPH is about tenfold higher than that of NADH in cyanobacteria [26], the 4:6 ratio was selected to optimize the in vitro system. 

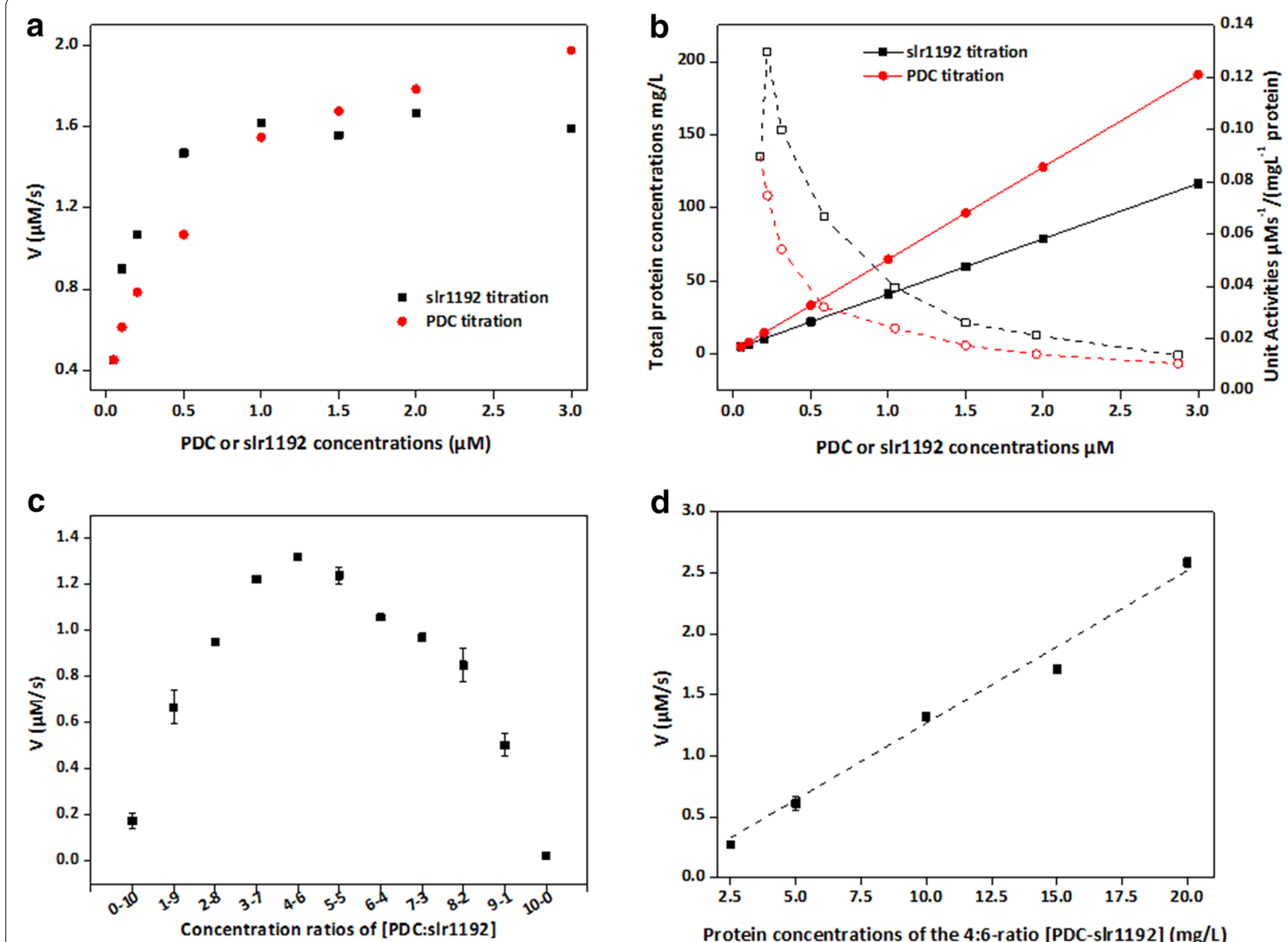

Protein concentrations of the 4:6-ratio [PDC-sir 1192] (mg/L)
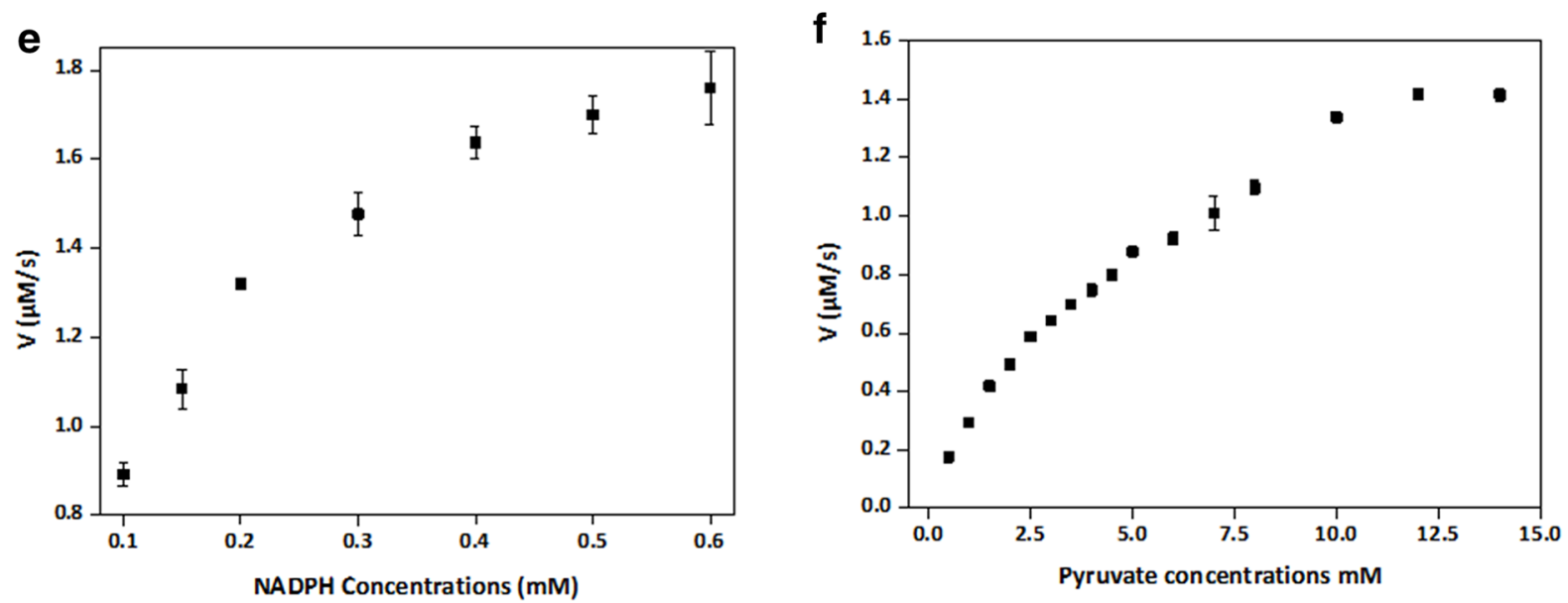

Fig. 2 Quantitative titration and optimization of the PDCzm-sIr1192 pathway based on the in vitro reconstitution system. a In slr 1192 titration system (black), PDCzm concentrations were fixed at $0.05 \mu \mathrm{M}$, while in PDCzm titration system (red), slr1192 concentrations were fixed at $0.05 \mu \mathrm{M}$. b Total protein concentrations (closed) and unit activities (open) dynamics of the PDCzm titration (circle) and slr1192 titration (square) systems. C Regulation of PDCzm and slr1192 ratios in the in vitro reconstitution systems with fixed total protein concentrations (10 mg/l). d Titration of the optimized (PDCzm-sIr1192) unit with a concentration ratio of 4:6. The single unit indicated a concentration ratio of 4:6 between PDCzm and slr1 192, while serial concentrations of such a unit were added to the in vitro system. e Titration of NADPH as cofactor in the optimized in vitro system. $\mathbf{f}$ Titration of pyruvate as substrate in the optimized in vitro system 
To evaluate the stability of the optimized concentration ratio, we added a series of concentrations of PDCzmslr1192 (4:6 ratio) into the in vitro system, and calculated the reaction velocity dynamics. Figure $2 \mathrm{~d}$ showed a nearly linear increase of reaction velocities when total protein concentrations increased from 2.5 to $20 \mathrm{mg} / \mathrm{l}$. These results indicated that as for cyanobacteria engineering for efficient synthesis of ethanol, concentrations of PDCzm and slr1192 should be regulated to be much higher and held to the ratio of about 4:6.

\section{Substrate, cofactors, and metal ions titrations in the optimized in vitro system}

The successful catalysis of PDCzm and slr1192 was assisted by several other components, including cofactor supply, substrate supply, and metal ions. So we further quantified contributions and influences of specific factor for the catalytic properties of the PDCzm-slr1192 pathway. As reported above, cofactor types (NADPH and NADH) greatly determined activities of slr1192. In this part, we performed in vitro titration to assess the concentration influence from $\mathrm{NADPH}$. Serial concentrations of NADPH, from 0.1 to $0.6 \mathrm{mM}$, were added to the in vitro system with $10 \mathrm{mg} / \mathrm{l}$ PDCzm-slr1192 (4:6 ratio as previously optimized), and the velocity dynamics versus NADPH concentrations were fitted with a Michaelis-Menten model as applied for enzyme titrations (Fig. 2e). The $K_{m}$ of NADPH for titration in the $i n$ vitro system was $0.136 \mathrm{mM}$, with a $V_{\max }$ value of $2.211 \mu \mathrm{M} / \mathrm{s}$.

Pyruvate supply seemed to be of great importance for catalytic properties of the PDCzm-slr1192 pathway. The velocities would be gradually improved to the maximal level when pyruvate concentrations were above $10 \mathrm{mM}$ (Fig. 2f), a level much higher than the actual pyruvate concentrations in cyanobacteria cell (about 20-100 $\mu \mathrm{M}$ ) $[7,27] . K_{m}$ and $V_{\max }$ values calculated from the pyruvate titration assay were $6.496 \mathrm{mM}$ and $2.064 \mu \mathrm{M} / \mathrm{s}$, respectively (Table 1).

Table 1 In vitro titration fit values of PDCzm, slr1192, NADPH, pyruvate, TPP, $\mathbf{M g}^{2+}$ ion and acetaldehyde

\begin{tabular}{lll}
\hline Components & $\boldsymbol{K}_{\mathbf{5 0}} \boldsymbol{K}_{\boldsymbol{m}}$ & $\boldsymbol{V}_{\boldsymbol{m a x}}(\boldsymbol{\mu M} \mathbf{s})$ \\
\hline PDCzm & $0.326 \pm 0.071 \mu \mathrm{M}$ & $2.074 \pm 0.120$ \\
slr1192 & $0.109 \pm 0.014 \mu \mathrm{M}$ & $1.722 \pm 0.045$ \\
NADPH & $0.136 \pm 0.013 \mathrm{mM}$ & $2.211 \pm 0.085$ \\
Pyruvate & $6.496 \pm 0.505 \mathrm{mM}$ & $2.064 \pm 0.104$ \\
TPP & $0.011 \pm 0.002 \mathrm{mM}$ & $1.405 \pm 0.054$ \\
Mg & $0.104 \pm 0.017 \mathrm{mM}$ & $1.329 \pm 0.043$ \\
Acetaldehyde & $0.393 \pm 0.130 \mathrm{mM}$ & $3.538 \pm 0.228$ \\
\hline
\end{tabular}

PDCzm and slr1192 titrations were performed in an in vitro system containing $10 \mathrm{mM}$ pyruvates, $0.2 \mathrm{mM}$ NADPH, $10 \mathrm{mM} \mathrm{MgCl}, 0.1 \mathrm{mM} \mathrm{TPP}, 0.05 \mu \mathrm{M}$ PDCzm or slr1192. NADPH, pyruvate, TPP, $\mathrm{Mg}^{2+}$ and acetaldehyde titrations were performed in an optimized system containing $10 \mathrm{mg} / \mathrm{l} \mathrm{PDCzm}$-s/r1192 with a concentration ratio of $4: 6$
We also regulated concentrations of acetaldehyde, the process metabolite, TPP and $\mathrm{Mg}^{2+}$, another two important factors regulating properties of PDCzm, and calculated the activities dynamics (Additional file 1: Figures S2 and S3). As shown in Table $1, K_{m}$ values of acetaldehyde, TPP and $\mathrm{Mg}^{2+}$ were 0.393, 0.011 and $0.104 \mathrm{mM}$, respectively.

\section{Genetic engineering of Synechocystis for regulation of PDCzm-sIr1192 ratios}

In vitro reconstitution and quantitative titrations of PDCzm and slr1192 indicated that conversion process from pyruvate to acetaldehyde was the rate-limiting step of the ethanol synthesis pathway, thus increasing the PDCzm-slr1192 concentration ratio should be a target for pathway optimization. For confirmation, we constructed Synechocystis strains with different PDCzm-slr1192 copy number ratios to generate diverse concentration ratios. Previously we have developed two Synechocystis mutant strains for ethanol synthesis, Syn-ZG25 (ZG25 hereafter) containing 1 PrbcL-PDC-slr1192 cassette copy and SynHZ24 (HZ24 hereafter) containing 2 PrbcL-PDC-slr1192 cassette copies. In vivo concentrations of PDCzm and slr1192 were both significantly improved in HZ24 comparing with ZG25, while the concentration ratio was relatively constant [18]. To disturb the PDCzm-slr1192 concentration ratio, we constructed a strain containing 1 PrbcL-PDC-slr1192 copy (on the slr0168 site, a neutral site on chromosome of Synechocystis sp. PCC6803) and 1 PrbcL-PDC copy (on the slr1993-slr1994 site, encoding acetyl CoA acetyltransferase and 3-ketoacyl-ACP reductase, essential for PHB synthesis in Synechocystis sp. PCC6803, slr9394 hereafter). The new strain was developed from ZG25, and termed as Syn-YQ4 (YQ4 hereafter), as shown in Fig. 3a.

Western blot analysis (Fig. 3b) revealed that comparing with ZG25 and HZ24, PDCzm-slr1192 concentration ratio in YQ4 was significantly improved. Slr1192 concentrations in YQ4 and ZG25 were on the same level, much lower than that of HZ24, while PDCzm concentration in YQ4 was even higher than that in HZ24 (as shown in Additional file 1: Figure S4).

Catalytic activities calculations further confirmed the results of protein concentration analysis. As shown in Fig. 3c, alcohol dehydrogenase activities of HZ24 crude enzyme was $4.76 \mu \mathrm{mol} / \mathrm{s} / \mathrm{g}$, about $40 \%$ higher than that of ZG25 and YQ4 (3.39 and $3.41 \mu \mathrm{mol} / \mathrm{s} / \mathrm{g}$, respectively). While activities of pyruvate decarboxylase in HZ24 and YQ4 crude enzymes were quite close to each other (1.28 and $1.41 \mu \mathrm{mol} / \mathrm{s} / \mathrm{g}$, respectively), more than twofold than that of ZG25 $(0.55 \mu \mathrm{mol} / \mathrm{s} / \mathrm{g})$. PDCzm activity patterns matched well with the PDCzm-slr1192 pathway activity analyses, velocities of HZ24 and YQ4 crude enzymes 

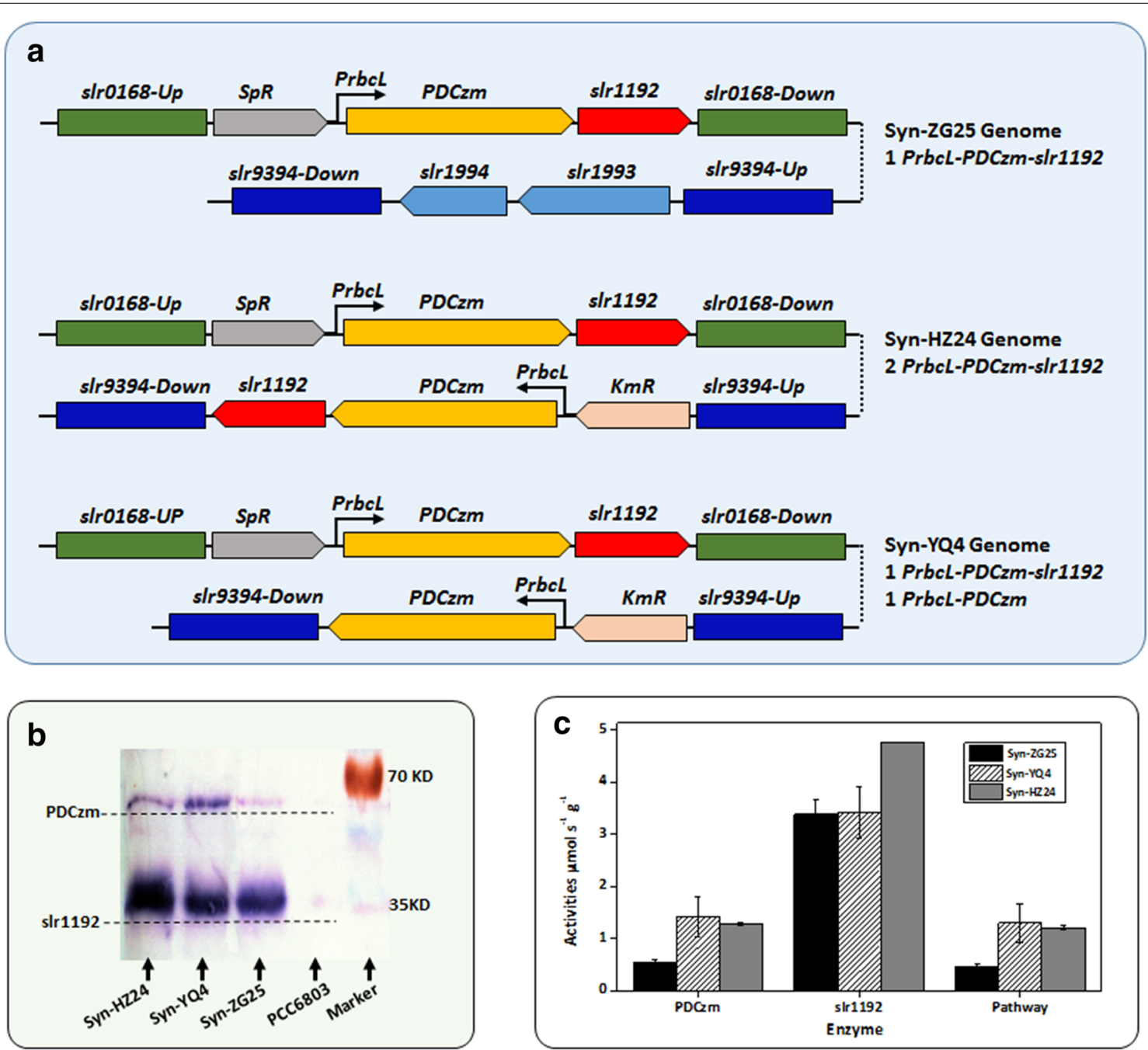

Fig. 3 Genetic engineering of Synechocystis for regulation of PDCZm-slr1192 ratios. a Genetic modifications on genomes of Syn-ZG25, Syn-HZ24, and Syn-YQ4. Slr0168-UP and slr0168-Down up-stream and down-stream homologous regions for PrbcL-PDCZm-slr1192 cassette integration with slr0168 site. Slr9394-Up and slr9394-Down up-stream and down-stream homologous regions for PrbcL-PDCzm-slr1192 cassette integration with slr1993-sIr1994 site. SpR spectinomycin resistance gene. KmR kanamycin resistance gene. b Western blot analysis for PDCzm and slr1192 concentration in PCC6803, Syn-ZG25, Syn-HZ24, and Syn-YQ4. c Pyruvate decarboxylase, alcohol dehydrogenase, and PDCzm-slr1192 pathways activities calculations for crude enzyme of Syn-ZG25, Syn-HZ24, and Syn-YQ4

on the same level (1.29 and $1.30 \mu \mathrm{mol} / \mathrm{s} / \mathrm{g}$, respectively), while much higher than that of ZG25 $(0.46 \mu \mathrm{mol} / \mathrm{s} / \mathrm{g})$.

For accurate evaluation of ethanol synthesis capacities of the Synechocystis mutants holding diverse PDCzmslr1192 ratios and concentrations, we cultivated the strains in BG11 medium using $50 \mathrm{mM} \mathrm{NaHCO}$ as sole carbon source. As shown in Fig. 4, in 2-day cultivation utilizing $\mathrm{NaHCO}_{3}$, over $20 \%$ of the fixed carbon was converted into ethanol in HZ24 and YQ4, while in ZG25 the ratio was about $10 \%$. Ethanol concentrations in cultures of ZG25, YQ4, and HZ24 were 0.093, 0.153, and $0.15 \mathrm{~g} / \mathrm{l}$, respectively. Ethanol synthesis and carbon partitioning ratios indicated that YQ4 strain obtained the same level of ethanol-producing capacities comparing with HZ24, while with much lower slr1192 concentrations and activities.

Growth and ethanol synthesis capacities of the Synechocystis mutants holding diverse PDCzm-slr1192 ratios and concentrations were also evaluated with a column photo-reactor system as previously introduced [18]. As shown in Fig. 4b, c, after 9 days of cultivation, ethanol concentrations of the YQ4 and HZ24 cultivation system reached 2.2-2.3 g/l, while that of the ZG25 system was $1.2 \mathrm{~g} / \mathrm{l}$. Ethanol production of the YQ4 strain was improved by twofold than that of ZG25. 

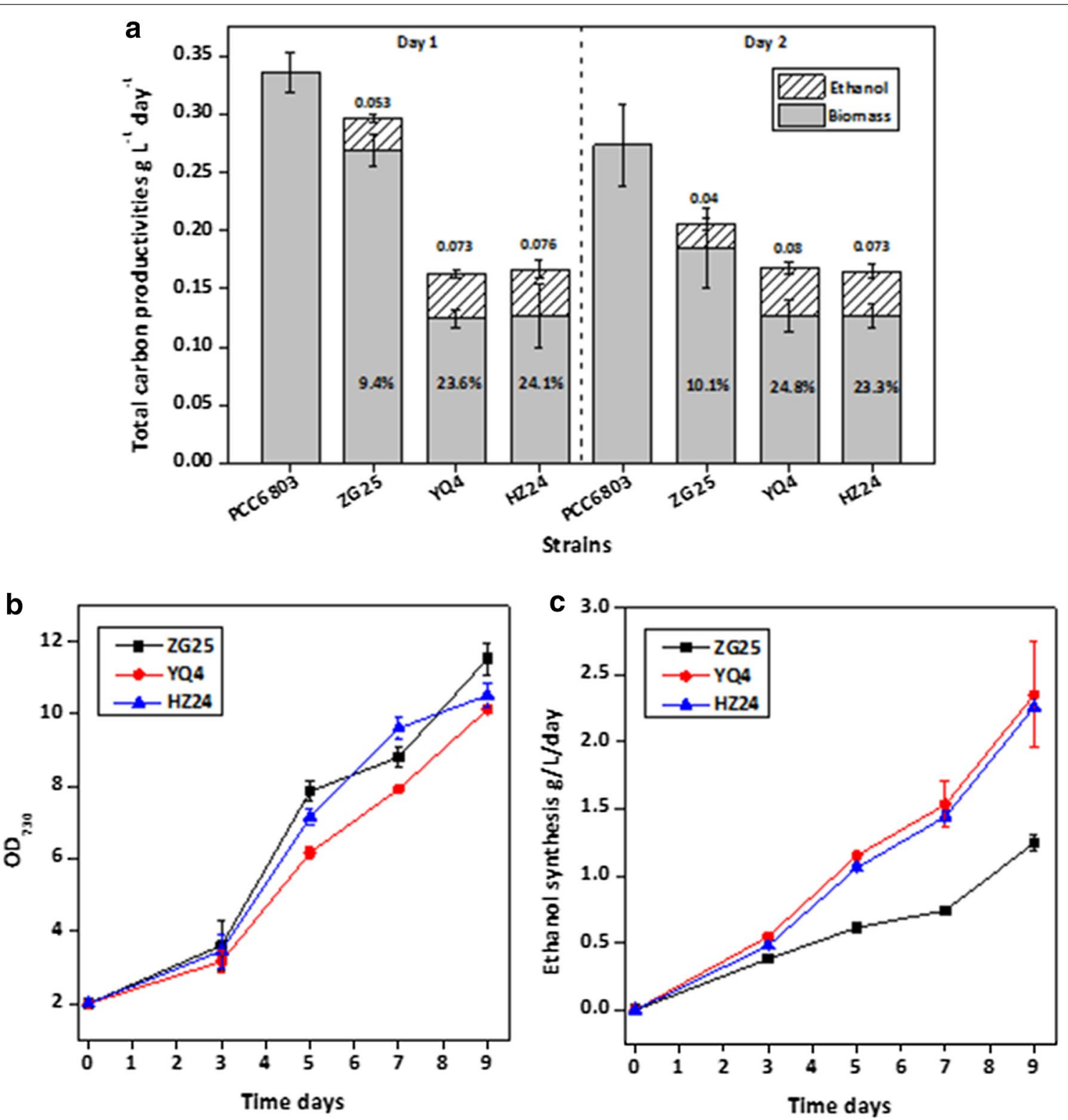

Fig. 4 Growth and ethanol production assay of strains with diverse PDCzm-sIr1192 ratios. a Total carbon productivities and carbon partitioning ratio to ethanol of PCC6803, Syn-ZG25, Syn-HZ24, and Syn-YQ4, taking $\mathrm{NaHCO}_{3}$ as carbon source. Carbon distributed to ethanol and biomass was calculated by measurement of ethanol concentration and OD730. Numbers in the bars percent of total fixed carbon distributed to ethanol, numbers above the bars the actual ethanol concentration synthesized by the strain ( $\mathrm{g} / \mathrm{l} /$ day). $\mathbf{b}$ Growth and $\mathbf{c}$ ethanol production of the three strains cultivated in column photo-reactor system

\section{Pyruvate-feeding assay of YQ4 strain}

As indicated by the in vitro titration results, improving pyruvate supply would benefit the ethanol generation capacities of engineered Synechocystis strains. Previously it has been discovered that environmental pyruvate could be taken up and metabolized by cyanobacterium Synechococcus elongatus PCC7942 [28], thus we applied a convenient strategy to evaluate the influence of increased pyruvate supply by adding pyruvate to the BG11 medium and calculate the biomass and ethanol accumulation.

As shown in Fig. 5, when $50 \mathrm{mM}$ pyruvate was added into BG11 medium to replace $\mathrm{NaHCO}_{3}$ as sole carbon source, carbon partitioning ratio of ethanol in YQ4 strain was sharply improved to $41.2 \%$, while both biomass and ethanol accumulations were significantly inhibited. When $25 \mathrm{mM}$ pyruvate and $50 \mathrm{mM} \mathrm{NaHCO}$ 

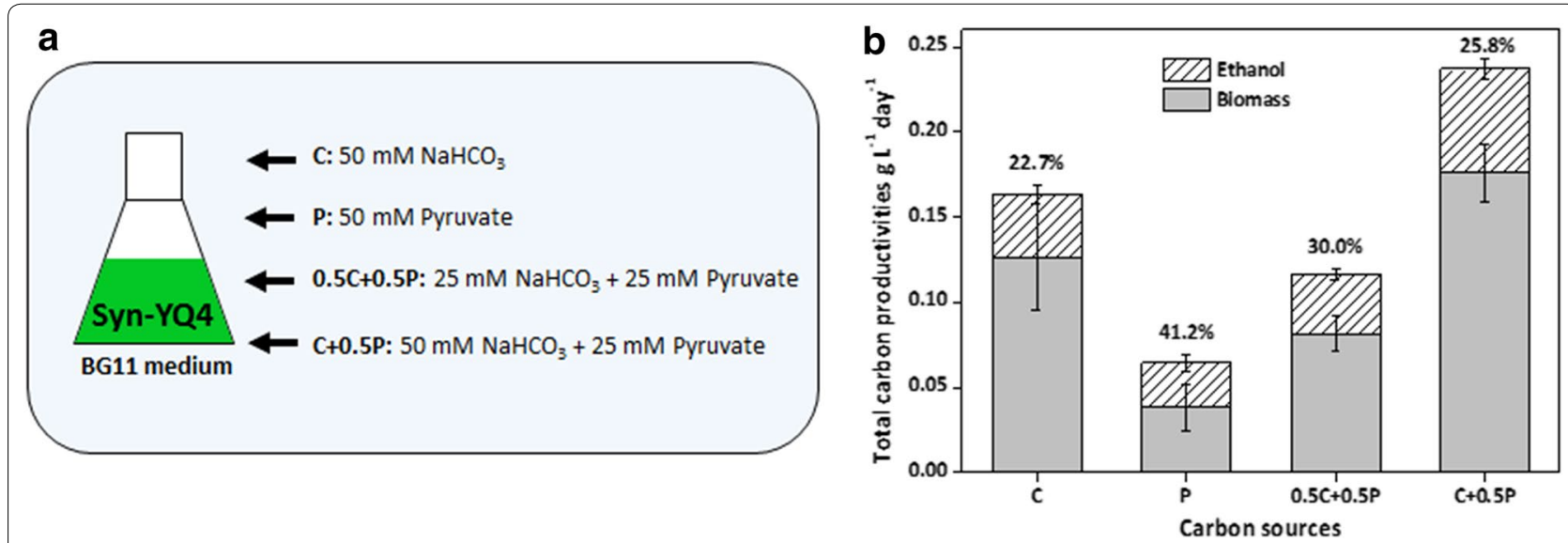

Fig. 5 Total carbon productivities and carbon partitioning ratio to ethanol of Syn-YQ4 in BG11 medium supplemented with pyruvate. a Concentrations of $\mathrm{NaHCO}_{3}$ and pyruvate for different feeding treatments. $\mathbf{b}$ Carbon accumulated in biomass and ethanol for YQ4 treated with different feeding pattern. Numbers above the bars the percent of total carbon distributed to ethanol

were simultaneously supplemented into BG11, speeds of biomass accumulation and ethanol synthesis were both improved, with an increased carbon partitioning ratio to ethanol. Results of the YQ4 strain pyruvate-feeding cultivation indicated that redistributing more carbon fixed by cyanobacteria to pyruvate might be useful strategy for enhancing ethanol productivities.

\section{Discussion}

As one of the most attractive and promising technical routes for biofuels production, exploring metabolic engineering principles for cyanobacteria synthesis of ethanol is of great demonstrative significance for development of efficient photosynthetic cell factories. Previously it has been proved that as for cyanobacteria-based production of chemicals and biofuels, metabolic capacities of the core pathways usually imposed the essential control over the product formation rates and carbon partitioning ratios $[2,11,18,29,30]$. Focusing on ethanol synthesis in cyanobacteria, the most effective improvement was achieved by increasing copy numbers of PDCzm-slr1192 on chromosomes or high-copy plasmids [18, 19], further confirming that the metabolic pathway activity was the main restricting factor for photosynthetic production of ethanol. However, expression strengths of the catalytic components could not be endlessly increased due to the limitation of genetic manipulation tools and the requirements of the host cells for maintaining basic homeostasis. Thus economical, balanced and efficient metabolic pathways were premises for development of more powerful photosynthetic cellular factories, which requires comprehensive understanding of the catalytic processes. In this work, we provided a paradigm for systematically characterizing and optimizing an essential ethanol synthesis metabolic pathway of engineered cyanobacteria strains, combining in vitro reconstitution, genetic engineering and feeding-cultivation.

In vitro reconstitution and steady-state analysis is a newly arising strategy for monitoring and unveiling the kinetic characteristics and system properties of metabolic pathways. The information thus gained has facilitated understanding and optimizing E. coli fatty acid synthase system [31], farnesene production pathway [32], and cyanobacteria fatty acid synthase system [24]. Comparing with previous in vitro reconstitution research, we attempted to optimize enzyme ratios with a constant total concentrations, rather than simply single-factor titration. In our previously developed ethanol synthesis strains ZG25 and HZ24, the in vivo concentration ratios of PDCzm to slr1192 were about 1:30-1:40 [18], far away from the most economical ratio of 1:2.5 we identified in this work, indicating that PDCzm activities hold the control over ethanol generation performance. For validation, we constructed a new Synechocystis strain YQ4 based on ZG25, with improved PDCzm concentrations. Biomass accumulation and ethanol generation assays revealed that the ethanol synthesis capacities of YQ4 were significantly improved than that of ZG25, while totally comparable with that of HZ24, although the slr1192 concentration was significantly decreased. Information gained from in vitro reconstitution and genetic engineering indicated that further enhancing PDCzm concentrations or activities should be the key point for enabling more efficient photosynthetic production of ethanol, which would be benefited from the development of novel expression toolkit in cyanobacteria [33, 34]. Protein engineering should be an alternative strategy. Considering that pyruvate decarboxylase from $Z$. mobilis had the highest 
reported catalytic activities [35], equipping the cell factory with optimized PDCZm obtained from directed evolution or rational designing might be of great significance for accelerating the pyruvate decarboxylation process during ethanol synthesis.

With removed restrictions from metabolic enzymes, reaction velocities of the in vitro systems were mainly controlled by substrate supply. As for $10 \mathrm{mg} / \mathrm{l} \mathrm{PDCzm-}$ slr1192, the reaction velocities would be maximized when initial pyruvate concentrations were above $10 \mathrm{mM}$, a level far more than normal concentration in cyanobacteria (about 20-100 $\mu \mathrm{M}$ ) [7, 27]. In order to explore the influence of increased in vivo supply of pyruvate, we added pyruvate into culture medium of the newly obtained Synecgocystis strain YQ4. As expected, when pyruvate was added, carbon partitioning ratio of ethanol was sharply increased. However, it is also noteworthy that when pyruvate concentration reached a high level, both cell growth and ethanol synthesis were significantly inhibited, indicating that a balance should be maintained between the carbon source distributed to biomass and chemicals. Pyruvate is an essential cellular central metabolite in cyanobacteria, from which carbon flow was distributed to diverse derivates. Introduction of artificially designed metabolic modulars or pathways oriented with pyruvate have enabled production of diverse chemicals, including lactic acids [7], ethanol [8], 2,3-butanediol [36, 37], acetone [13], isopropanol [10], and butanol [9]. Concentration of the main precursor phosphoenolpyruvate (PEP) was about tenfold higher than that of pyruvate, thus enhancement of conversion from PEP to pyruvate might greatly benefit ethanol production as reported in lactic acid production engineering [27, 29]. Previously it has been proved that knockout of the glycogen synthesis pathway could effectively promote accumulation and excretion of pyruvate [38], thus systematic genetic modifications of cyanobacteria to rewire carbon flow for enhanced pyruvate supply should also be a promising strategy for development of more efficient photosynthetic microbial platforms.

Sufficient and suitable cofactor supply was always a guarantee for the unencumbered chemical production in cyanobacteria [29, 39]. Comparing with the initial photosynthetic cell factory for ethanol production, replacing the NADH-dependent AdhII from Z . mobilis with the native NADPH-dependent gene slr1192 significantly improved catalytic activities of the whole pathway [18]. In our in vitro titration assays with serial PDCzm-sir1192 concentration ratios from 1:9-7:3, reaction velocities were decreased by over $80 \%$ when taking NADH as cofactor rather than NADPH, indicating the importance of optimizing cofactor preferences in heterogeneous metabolic pathway of cyanobacteria. As for cyanobacteria, photosynthesis played an important role for guaranteeing the rapid turnover from $\mathrm{NADP}^{+}$to $\mathrm{NADPH}$, thus possibly maintaining the NADPH concentration at a favorable level for ethanol production. Engineering or updating the key components or enzymes in photosystem for more efficient supply of NADPH and carbon source could also contribute a lot for biosynthesis in cyanobacteria [40].

\section{Conclusions}

In summary, we combined in vitro reconstitution, genetic engineering, and feeding-cultivation for systematically characterizing and optimizing the PDCzmslr1192 pathways in engineered cyanobacteria strains. The information gained from in vitro monitoring and genetic engineering indicated that enhancing PDCzm activities and improving PDCzm-slr1192 concentration ratio to about 1:1.5 should be essential point for further improvement of ethanol generation rates. Taking the actual metabolite concentrations of cyanobacteria cells into consideration, expanding pyruvate supply might also benefit ethanol formation capacities in cyanobacteria.

\section{Methods}

\section{Chemicals and reagents}

Unless noted otherwise, all reagents were purchased from Sigma-Aldrich (USA). Taq DNA polymerase and all restriction enzymes were purchased from Fermentas (Canada) or Takara (Japan). The kits used for molecular cloning were from Omega (USA) or Takara (Japan). Oligonucleotides were synthesized and DNA sequencing was performed by Sunnybio (Shanghai, China).

\section{Strains and plasmids constructions}

Escherichia coli DH5 $\alpha$ was used in this work for plasmid construction and E. coli BL21 (DE3) was used as the host for protein expression. Strains Syn-ZG25 and SynHZ24 were constructed in our previous work [18], and Syn-YQ4 was constructed by introduction of the plasmid pYQ4 into the slr9394 site of the strain Syn-ZG25.

For construction of pYQ4 plasmid, up and down homologous recombination arms of slr9394 were cloned and fused with CK2 (kanamycin resistance gene) and PrbcL-PDC fragment, respectively, by overlap-PCR. The two fused fragments were finally inserted into pMD18T (TAKARA).

Transformation of plasmid pYQ4 into Syn-ZG25 was performed as the published procedures [41]. Syn-ZG25 was cultivated to exponential phase, and the cells were centrifuged, washed twice with fresh BG11 medium, and finally resuspended to a density of about $1 \times 10^{9} \mathrm{cells} / \mathrm{ml}$. Plasmid DNA was added to the cell suspension to a final concentration of about $10 \mu \mathrm{g} / \mathrm{ml}$. The cell-DNA mixture was incubated for $6 \mathrm{~h}$ at $30{ }^{\circ} \mathrm{C}$ under luminous intensity 
of about $50 \mu \mathrm{E} / \mathrm{m}^{2} / \mathrm{s}$ ) and then spread on nitrocellulose filters on BG11 agar plates for $24 \mathrm{~h}$. Finally, the filters were moved to fresh BG11 agar plates containing $25 \mu \mathrm{g} /$ $\mathrm{ml}$ kanamycin and $25 \mu \mathrm{g} / \mathrm{ml}$ spectinomycin. After cultivation for about 10 days, single colonies were separated and cultivated in liquid BG11 for re-checking.

\section{Protein expression and purification}

Plasmid pXT4 for PDCzm expression and plasmid pXT113A for slr1192 expression were constructed in previous work [18]. E. coli BL21 (DE3) was transformed with pXT4 and pXT113A, respectively. One single colony of the transformed E. coli BL21 (DE3) strain was inoculated in $11 \mathrm{ml} \mathrm{LB}$ medium added with $50 \mu \mathrm{g} / \mathrm{ml}$ kanamycin and cultivated for $12 \mathrm{~h}$ before being inoculated into 11 super broth (tryptone $1.2 \%$, yeast extract $1.4 \%$, $\mathrm{KH}_{2} \mathrm{PO}_{4} 0.38 \%, \mathrm{~K}_{2} \mathrm{HPO}_{4} 1.25 \%$, glycerol $0.5 \%$ ). The broth was cultivated at $37{ }^{\circ} \mathrm{C}, 220 \mathrm{rpm}$ for about $2.5 \mathrm{~h}$, till the $\mathrm{OD}_{600}$ reached 0.4 and isopropyl-D-thiogalactopyranoside (IPTG) was added to a final concentration of $0.5 \mathrm{mM}$. The culture is incubated at $16{ }^{\circ} \mathrm{C}$ overnight with shaking at $180 \mathrm{rpm}$.

Cells were harvested by centrifugation, resuspended in binding buffer $(20 \mathrm{mM}$ Tris- $\mathrm{HCl}, 0.5 \mathrm{M} \mathrm{NaCl}, \mathrm{pH} 7.9$ for slr1192 and $\mathrm{pH} 7.5$ for PDCzm) and lysed by sonication. The cell lysate was pelleted by centrifugation at $12,000 \mathrm{~g}, 4^{\circ} \mathrm{C}$ for $30 \mathrm{~min}$. The supernatant was immediately blended with Ni-NTA resin (Novagen), pre-equilibrated with the binding buffer, and agitated at $4{ }^{\circ} \mathrm{C}$ for $1 \mathrm{~h}$. The equilibrated system was then transferred into a $5-\mathrm{ml}$ column that was washed sequentially with $25 \mathrm{ml}$ binding buffer, $25 \mathrm{ml}$ washing buffer containing serial concentrations $(20,40$, and $80 \mathrm{mM})$ of imidazole to remove nonspecifically bound proteins, and then $25 \mathrm{ml}$ of the washing buffer (containing $100 \mathrm{mM}$ imidazole for PDCzm and $250 \mathrm{mM}$ for slr1192) to elute the target proteins. The eluted protein was examined by SDS-PAGE and the elution fractions with desired purity were pooled, desalinated with $100 \mathrm{mM}$ Tris- $\mathrm{HCl}, 0.5 \mathrm{M} \mathrm{NaCl}(\mathrm{pH} 7.5$ for PDCzm and pH 7.9 for slr1192), and finally concentrated with Amicon Ultra centrifugal filters (Milipore). Concentrations of the purified proteins were quantified using the Bradford method [42].

\section{In vitro reconstitution and UV kinetic assay of the PDCzm-sIr1192 pathway}

Initial rates of the ethanol synthesis pathway of PDCzmslr1192 was quantified using a spectrophotometric $\mathrm{NAD}(\mathrm{P}) \mathrm{H}$ consumption assay, as previously described for PDCzm activity in vitro assay [18], while the components and respective concentrations of the in vitro system were modified and regulated as required. Slr1192 oxidized one NADPH or NADH per turn over, so the $340 \mathrm{~nm}$ absorbance of the reaction system decreased during $\mathrm{NAD}(\mathrm{P}) \mathrm{H}$ to $\mathrm{NAD}(\mathrm{P})^{+}$could be directly related to ethanol synthesis.

For steady-state analysis, an initial in vitro system containing $100 \mathrm{mM}$ Tris- $\mathrm{HCl}, 10 \mathrm{mM} \mathrm{MgCl}_{2}, 0.1 \mathrm{mM}$ thiamine pyrophosphate (TPP), $0.05 \mu \mathrm{M}$ PDCzm, $0.05 \mu \mathrm{M}$ slr1192, $0.2 \mathrm{mM} \mathrm{NADPH}$, and $10 \mathrm{mM}$ pyruvate was constructed ( $\mathrm{pH} 7.5)$, and the total volume was $500 \mu \mathrm{l}$. Titrations of PDCzm and slr1192 were performed in the initial system, while for titration of other components, $4 \mathrm{mg} / \mathrm{l}$ PDCzm and $6 \mathrm{mg} / \mathrm{l}$ slr1192 were added to the reaction as introduced below. Reactions were always initiated by addition of the PDCzm-slr1192 components, and the $340 \mathrm{~nm}$ absorbance is measured with a Beckman-coulter DU-800 spectrophotometer. All experiments were repeated for at least 3 times to verify the trends and parametric reproducibility. For PDCzm-slr1192 pathway activities analysis, crude enzymes of the wild-type and engineered Synechocystis strains were added into the reaction system instead of purified enzymes.

\section{Cultivation of Synechocystis strains}

For growth and ethanol production assay, Synechocystis strains were cultivated in BG11 medium added with $50 \mathrm{mM} \mathrm{NaHCO}, 10 \mathrm{mM}$ TES-NaOH (pH 8.0), and appropriate antibiotics, and incubated with shaking at $30{ }^{\circ} \mathrm{C}$ in a incubator with $130 \mathrm{rpm}$ under moderate intensity white-light illumination (about $100 \mu \mathrm{E} / \mathrm{m}^{2} / \mathrm{s}$ ). Cell density and ethanol concentration of in the culture were calculated every $24 \mathrm{~h}$ by removing $0.5 \mathrm{ml}$ from the $25 \mathrm{ml}$ total volume. For pyruvate-feeding assay, $25 \mathrm{mM}$ pyruvate plus with $25 \mathrm{mM} \mathrm{NaHCO}$ or $50 \mathrm{mM}$ pyruvate is supplemented into the BG11 medium to replace the $50 \mathrm{mM} \mathrm{NaHCO}$.

Optical densities of the cyanobacteria culture were used for calculation of carbon in biomass. Dry cell weight (gDW) was calculated from OD730 using the value of $0.22 \mathrm{~g} \mathrm{DW} / \mathrm{l} / \mathrm{OD} 730$ [28], while carbon content of cells was taken as $51.34 \%[28,43]$. Carbon in ethanol was calculated from the concentration of ethanol from the culture. Partitioning ratio of carbon to ethanol was obtained by dividing the carbon in ethanol by the sum of total carbon distributed in ethanol and biomass during the same period.

Synechocystis mutant strains were also cultivated in column photo-bioreactors as previously introduced [18]. Synechocystis cells grown to the exponential phase were harvested by centrifugation and resuspended to an $\mathrm{OD}_{730}$ of 2.0 with $200 \mathrm{ml}$ fresh BG11 medium in column bioreactor $(580 \mathrm{~mm} \times 30 \mathrm{~mm})$ with a rubber plug. The cultures in the column photo-bioreactor system were sparged with $5 \% \mathrm{CO}_{2}$-air under $37{ }^{\circ} \mathrm{C}$ with constant $100 \mu \mathrm{E} / \mathrm{m}^{2} / \mathrm{s}$. The rate of gas addition is about $200 \mathrm{ml} /$ 
min. To measure the ethanol production more accurately, a condenser and a recovery bottle were connected with the column bioreactor due to the volatility of ethanol [18].

\section{Extraction of Synechocystis cell crude enzyme}

$20 \mathrm{ml}$ Synechocystis culture at late exponential growth with an OD730 of about 1.5 was harvested by centrifugation at $4{ }^{\circ} \mathrm{C}$. The resulted cell pellets were resuspended in $2 \mathrm{ml}$ pre-chilled Tris- $\mathrm{HCl}$ buffer $(50 \mathrm{mM}$ Tris- $\mathrm{HCl}$, $\mathrm{pH}$ 8.0) and disrupted with $100 \mu \mathrm{m}$ glass beads (Sigma). After removing the cell debris and glass beads by $4{ }^{\circ} \mathrm{C}$ centrifugation, the supernatants were collected for western blot analysis and crude enzyme activity assay. Protein concentrations of the cell-free extracts were also measured with Bradford method [42].

\section{Crude enzyme activity assay}

Alcohol dehydrogenase and pyruvate decarboxylase activities of the Synechocystis mutants cell crude enzymes were analyzed as previously introduced with minor modifications [18]. For alcohol dehydrogenase activity analysis, crude enzyme was added to the reaction buffer containing $100 \mathrm{mM}$ Tris- $\mathrm{HCl}, 0.2 \mathrm{mM}$ $\mathrm{NADPH}$, and $10 \mathrm{mM}$ acetaldehyde. For pyruvate decarboxylase activity analysis, crude enzyme were added to the reaction buffer containing $100 \mathrm{mM}$ Tris $-\mathrm{HCl}$, $10 \mathrm{mM} \mathrm{MgCl}, 0.1 \mathrm{mM}$ thiamine pyrophosphate (TPP), $0.2 \mathrm{mM} N A D P H, 10 \mathrm{mM}$ pyruvate and $400 \mu \mathrm{M}$ purified slr1192. Reactions were always initiated by addition of crude enzymes, and the $340 \mathrm{~nm}$ absorbance is measured with a Beckman-coulter DU-800 spectrophotometer. All experiments were repeated for at least 3 times to verify the trends and parametric reproducibility.

\section{Ethanol production analysis}

Samples of the cyanobacteria culture were centrifuged at $10,000 \mathrm{~g}$ for $10 \mathrm{~min}$, and the supernatants were used for ethanol concentration measurement with an SBA40c biosensor analyzer (Shandong Academy of Sciences, China) equipped with ethanol oxidase immobilized membrane, as previously introduced [18].

\section{SDS-PAGE and western blot analysis}

The protein samples were analyzed on $12 \%$ SDS-PAGE with a standard procedure and blotted to PVDF membrane, sealed in $5 \%$ nonfat milk-TBST buffer (TBS added with $0.05 \%$ Tween-20) at $4{ }^{\circ} \mathrm{C}$ overnight. First, the membrane was incubated with anti- $6 \times$ His-Tag monoclonal antibodies (from mouse) for $3 \mathrm{~h}$ and washed 3 times with TBST (15 min each time). Second, the membrane were incubated with an alkaline phosphatase-linked secondary antibody (donkey anti-mouse) for $1 \mathrm{~h}$ and washed 3 times with TBST (15 min each time). Finally, the membrane was colored with BCIP/NBT (Sigma). For expression level comparisons, the results of western blot were calculated with ImageJ.

\section{Additional file}

Additional file 1: Figure S1. Regulation of PDCzm and slr1192 ratios in the in vitro reconstitution systems with fixed total protein concentrations $(10 \mathrm{mg} / \mathrm{L})$ taking $\mathrm{NADH}$ as cofactor. Figure S2. Titrations of TPP and $\mathrm{Mg}^{2+}$ for PDCzm-slr1192 pathway in the optimized in vitro reconstitution system containing total protein concentrations ( $10 \mathrm{mg} / \mathrm{L}$ ) taking NADPH as cofactor. Figure S3. Titration of acetaldehyde for PDCzm-sIr1192 pathway in the optimized in vitro reconstitution system containing the total protein concentration (10 mg/L) taking NADPH as cofactor. Figure S4. Relative expression level of PDCzm and slr1192 in Syn-ZG25, Syn-YQ4 and SynHZ24. The concentration of specific protein in ZG25 was set as 100.

\section{Abbreviations}

PDC: pyruvate decarboxylase; PDCzm: pyruvate decarboxylase from Zymomonas mobilis; NADPH: reduced nicotinamide adenine dinucleotide phosphate; NADP: nicotinamide adenine dinucleotide phosphate; NADH: reduced nicotinamide adenine dinucleotide; NAD: nicotinamide adenine dinucleotide; TPP: thiamine pyrophosphate; SpR: spectinomycin resistance gene; KmR: kanamycin resistance gene; slr9394: slr1993 and slr1994.

\section{Authors' contributions}

GL designed the research, performed in vitro reconstitution, strains evaluations and enzymatic assays, analyzed the data and drafted the manuscript. YQ constructed the Syn-YQ4 strain, performed the column photo-reactor cultivations and helped to draft the manuscript. MW participated in strain constructions and evaluations, and helped to draft the manuscript. ZL participated in project design and in vitro reconstitution, and helped to revise the manuscript. YD participated in protein purification, enzyme activities assays and the manuscript drafting. XT participated in constructions of plasmid and Synechocystis mutant strains and the manuscript revising. $X \mathrm{~L}$ conceived and supervised the project, participated in data analysis and revised the manuscript. All authors read and approved the final manuscript.

\section{Author details}

${ }^{1}$ Key Laboratory of Biofuels, Qingdao Institute of Bioenergy and Bioprocess Technology, Chinese Academy of Sciences, No. 189 Songling Road, Qingdao 266101, China. ${ }^{2}$ Shandong Provincial Key Laboratory of Synthetic Biology, Qingdao Institute of Bioenergy and Bioprocess Technology, Chinese Academy of Sciences, No. 189 Songling Road, Qingdao 266101, China. ${ }^{3}$ Shandong Provincial Key Laboratory of Energy Genetics, Qingdao Institute of Bioenergy and Bioprocess Technology, Chinese Academy of Sciences, No. 189 Songling Road, Qingdao 266101, China. ${ }^{4}$ Qingdao University of Science and Technology, Qingdao 266061, China. ${ }^{5}$ University of Chinese Academy of Sciences, Beijing 100049, China. ${ }^{6}$ Present Address: College of Bioscience and Bioengineering, Jiangxi Agricultural University, Nanchang 330045, Jiangxi, China.

\section{Acknowledgements}

This work was supported by the National High-Tech Research and Development Program of China (2012AA052103), the Excellent Youth Award of the Shandong Natural Science Foundation (JQ201306 to X. Lu), the Shandong Taishan Scholarship (X. Lu), the Shandong Postdoctoral Innovation Project (G. Luan, Post-doctor No. 145071), Scientific Funds for Outstanding Young Scientists of Shandong Province (BS2014SW021 to G. Luan) and China Postdoctoral Science Foundation (G. Luan, 2015M570616).

\section{Competing interests}

The authors declare that they have no competing interests.

Received: 10 September 2015 Accepted: 28 October 2015

Published online: 21 November 2015 


\section{References}

1. Keasling JD, Chou H. Metabolic engineering delivers next-generation biofuels. Nat Biotechnol. 2008;26(3):298-9.

2. Desai SH, Atsumi S. Photosynthetic approaches to chemical biotechnology. Curr Opin Biotechnol. 2013;24(6):1031-6.

3. Hellingwerf KJ, de Mattos MJT. Alternative routes to biofuels: light-driven biofuel formation from $\mathrm{CO}_{2}$ and water based on the 'photanol'approach. J Biotechnol. 2009;142(1):87-90.

4. Blatti JL, Michaud J, Burkart MD. Engineering fatty acid biosynthesis in microalgae for sustainable biodiesel. Curr Opin Chem Biol. 2013;17(3):496-505.

5. Lu X. A perspective: photosynthetic production of fatty acid-based biofuels in genetically engineered cyanobacteria. Biotechnol Adv. 2010;28(6):742-6.

6. Angermayr SA, Hellingwerf KJ, Lindblad P, de Mattos MJT. Energy biotechnology with cyanobacteria. Curr Opin Biotechnol. 2009;20(3):257-63.

7. Angermayr SA, Paszota M, Hellingwerf KJ. Engineering a cyanobacterial cell factory for production of lactic acid. Appl Environ Microbiol. 2012;78(19):7098-106

8. Deng MD, Coleman JR. Ethanol synthesis by genetic engineering in cyanobacteria. Appl Environ Microbiol. 1999;65(2):523-8.

9. Lan El, Liao JC. Metabolic engineering of cyanobacteria for 1-butanol production from carbon dioxide. Metab Eng. 2011;13(4):353-63.

10. Kusakabe T, Tatsuke T, Tsuruno K, Hirokawa Y, Atsumi S, Liao JC, et al. Engineering a synthetic pathway in cyanobacteria for isopropanol production directly from carbon dioxide and light. Metab Eng. 2013;20:101-8.

11. Wang WH, Liu XF, Lu XF. Engineering cyanobacteria to improve photosynthetic production of alka(e)nes. Biotechnol Biofuels. 2013;6:69

12. Wang JX, Zhang XQ, Shi ML, Gao LJ, Niu XF, Te RG, et al. Metabolomic analysis of the salt-sensitive mutants reveals changes in amino acid and fatty acid composition important to long-term salt stress in Synechocystis sp PCC 6803. Funct Integr Genomic. 2014;14(2):431-40.

13. Zhou J, Zhang HF, Zhang YP, Li Y, Ma YH. Designing and creating a modularized synthetic pathway in cyanobacterium Synechocystis enables production of acetone from carbon dioxide. Metab Eng. 2012;14(4):394-400.

14. He MX, Wu B, Qin H, Ruan ZY, Tan FR, Wang JL, et al. Zymomonas mobilis: a novel platform for future biorefineries. Biotechnol Biofuels. 2014;7:101.

15. Yomano LP, York SW, Zhou S, Shanmugam KT, Ingram LO. Reengineering Escherichia coli for ethanol production. Biotechnol Lett. 2008:30(12):2097-103.

16. Nikel PI, de Lorenzo V. Robustness of Pseudomonas putida KT2440 as a host for ethanol biosynthesis. New Biotechnol. 2014;31(6):562-71.

17. Vidal R, Lopez-Maury L, Guerrero MG, Florencio FJ. Characterization of an alcohol dehydrogenase from the Cyanobacterium Synechocystis sp. strain PCC 6803 that responds to environmental stress conditions via the Hik34-Rre1 two-component system. J Bacteriol. 2009;191(13):4383-91.

18. Gao ZX, Zhao H, Li ZM, Tan XM, Lu XF. Photosynthetic production of ethanol from carbon dioxide in genetically engineered cyanobacteria. Energy Environ Sci. 2012;5(12):9857-65.

19. Dienst D, Georg J, Abts T, Jakorew L, Kuchmina E, Borner T, et al. Transcriptomic response to prolonged ethanol production in the cyanobacterium Synechocystis sp PCC6803. Biotechnol Biofuels. 2014;7:21.

20. Dexter J, Fu PC. Metabolic engineering of cyanobacteria for ethanol production. Energy Environ Sci. 2009;2(8):857-64.

21. Yoshikawa K, Hirasawa T, Shimizu H. Effect of malic enzyme on ethanol production by Synechocystis sp. PCC 6803. J Biosci Bioeng 2014;119(1):82-4.

22. Dexter J, Armshaw P, Sheahan C, Pembroke JT. The state of autotrophic ethanol production in Cyanobacteria. J Appl Microbiol. 2015;119(1):11-24.

23. Wang JX, Chen L, Huang SQ, Liu J, Ren XY, Tian XX, et al. RNA-seq based identification and mutant validation of gene targets related to ethanol resistance in cyanobacterial Synechocystis sp PCC 6803. Biotechnol Biofuels. 2012:5:89
24. Kuo J, Khosla C. The initiation ketosynthase $(\mathrm{FabH})$ is the sole rate-limiting enzyme of the fatty acid synthase of Synechococcus sp. PCC 7002. Metab Eng. 2014;22:53-9.

25. Vidal R, Lopez-Maury L, Guerrero MG, Florencio FJ. Characterization of an alcohol dehydrogenase from the cyanobacterium Synechocystis sp strain PCC 6803 that responds to environmental stress conditions via the Hik34Rre1 two-component system. J Bacteriol. 2009;191(13):4383-91.

26. Cooley JW, Vermaas WF. Succinate dehydrogenase and other respiratory pathways in thylakoid membranes of Synechocystis sp. strain PCC 6803: capacity comparisons and physiological function. J Bacteriol. 2001;183(14):4251-8.

27. Takahashi H, Uchimiya H, Hihara Y. Difference in metabolite levels between photoautotrophic and photomixotrophic cultures of Synechocystis sp PCC 6803 examined by capillary electrophoresis electrospray ionization mass spectrometry. J Exp Bot. 2008;59(11):3009-18.

28. Oliver JWK, Atsumi S. A carbon sink pathway increases carbon productivity in cyanobacteria. Metab Eng. 2015;29:106-12.

29. Angermayr $S A$, van der Woude AD, Correddu D, Vreugdenhil A, Verrone $V$, Hellingwerf KJ. Exploring metabolic engineering design principles for the photosynthetic production of lactic acid by Synechocystis sp. PCC6803. Biotechnol Biofuels. 2014;7:99.

30. Angermayr SA, Hellingwerf KJ. On the use of metabolic control analysis in the optimization of cyanobacterial biosolar cell factories. J Phys Chem B. 2013:117(38):11169-75.

31. Yu X, Liu T, Zhu F, Khosla C. In vitro reconstitution and steady-state analysis of the fatty acid synthase from Escherichia coli. Proc Natl Acad Sci USA. 2011;108(46):18643-8.

32. Zhu F, Zhong X, Hu M, Lu L, Deng Z, Liu T. In vitro reconstitution of mevalonate pathway and targeted engineering of farnesene overproduction in Escherichia coli. Biotechnol Bioeng. 2014;111(7):1396-405.

33. Zhou J, Zhang H, Meng H, Zhu Y, Bao G, Zhang Y, et al. Discovery of a super-strong promoter enables efficient production of heterologous proteins in cyanobacteria. Sci Rep. 2014:4:4500.

34. Sakai Y, Abe K, Nakashima S, Ellinger JJ, Ferri S, Sode K, et al. Scaffold-fused riboregulators for enhanced gene activation in Synechocystis sp. PCC 6803. Microbiologyopen. 2015;4(4):533-40.

35. Siegert P, McLeish MJ, Baumann M, Iding H, Kneen MM, Kenyon GL, et al Exchanging the substrate specificities of pyruvate decarboxylase from Zymomonas mobilis and benzoylformate decarboxylase from Pseudomonas putida. Protein Eng Des Sel. 2005;18(7):345-57.

36. Oliver JW, Machado IM, Yoneda H, Atsumi S. Cyanobacterial conversion of carbon dioxide to 2,3-butanediol. Proc Natl Acad Sci USA. 2013;110(4):1249-54.

37. Savakis PE, Angermayr SA, Hellingwerf KJ. Synthesis of 2,3-butanediol by Synechocystis sp PCC6803 via heterologous expression of a catabolic pathway from lactic acid- and enterobacteria. Metab Eng. 2013;20:121-30.

38. Carrieri D, Paddock T, Maness PC, Seibert M, Yu JP. Photo-catalytic conversion of carbon dioxide to organic acids by a recombinant cyanobacterium incapable of glycogen storage. Energy Environ Sci. 2012;5(11):9457-61.

39. Akhtar MK, Jones PR. Cofactor engineering for enhancing the flux of metabolic pathways. Front Bioeng Biotechnol. 2014;2:30.

40. Atsumi S, Higashide W, Liao JC. Direct photosynthetic recycling of carbon dioxide to isobutyraldehyde. Nat Biotechnol. 2009;27(12):1177-80.

41. Williams JGK. Construction of specific mutations in photosystem-II photosynthetic reaction center by genetic-engineering methods in Synechocystis-6803. Methods Enzymol. 1988;167:766-78.

42. Bradford MM. A rapid and sensitive method for the quantitation of microgram quantities of protein utilizing the principle of protein-dye binding. Anal Biochem. 1976;72:248-54.

43. Young JD, Shastri AA, Stephanopoulos G, Morgan JA. Mapping photoautotrophic metabolism with isotopically nonstationary C-13 flux analysis. Metab Eng. 2011;13(6):656-65. 